\title{
SEL1L Gene
}

National Cancer Institute

\section{Source}

National Cancer Institute. SEL1L Gene. NCI Thesaurus. Code C105007.

This gene is involved in protein degradation. 